\title{
Research on Improving Transient Stability of Power System with Distributed BESS and its Coordinated Control Method
}

\author{
Can $\mathrm{Su}^{1 *}$, Hao Zhou ${ }^{1}$, Peng Yan ${ }^{1}$, Liang Meng ${ }^{1}$, Ziwei Cheng ${ }^{1}$ \\ ${ }^{1}$ Electric Power Research Institute of State Grid Hebei Electric Power Company, Shijiazhuang, Hebei, 050000, China
}

\begin{abstract}
The feasibility of battery energy storage system (BESS) in ameliorating power system transient stability has been proved. However, the required BESS capacity is large, which leads to high price. Meanwhile, the improving effect is significantly affected by fault location and BESS location. To address the problem, a distributed configuration of BESS, with a coordinated control method, is proposed in this paper. Firstly, an independent double-closed loop decoupling controller is designed for each BESS, so that the BESS unit can realize decentralized control according to its local correlation information. Secondly, particle swarm optimization is applied to optimize the control parameters of the distributed controllers to achieve coordinated control. Simulation results show that the distributed BESS controllers optimized by particle swarm optimization can better coordinate with each other, and the transient stability of the system is significantly improved.
\end{abstract}

\section{Introduction}

Transient stability analysis has always been an important subject in power system stability research. With the rapid development of energy storage technology, energy storage components provide new research ideas for power system transient stability control, among which BESS is a typical representative [1]-[3]. Compared with traditional components, BESS has incomparable advantages in improving the transient stability of the power system. Relevant studies have confirmed the feasibility of applying BESS to improve the small disturbance stability [4]-[7] and transient stability [8], [9] of the power system. However, the single BESS capacity required to effectively improve the transient stability of the power system is relatively large, and the effect is obviously affected by fault location and BESS location

The above-mentioned problems can be solved by distributing multiple small-capacity BESSs. There are relatively few studies in distributed BESS, and the coordinated control method is not clear. If the interaction between the multiple BESS controllers is not considered, their distributed and independent operations may lead to incoordination, even deteriorates the stability of the power system. Therefore, traditional coordinated control methods cannot be simply transplanted to the decentralized control of BESS.

In this paper, a double closed-loop controller is designed for each BESS [9]. Furthermore, particle swarm optimization is used to optimize the parameters of distributed controllers with multiple constraints to ensure the coordination between controllers. Simulation results show that the coordinated control method proposed in this paper is suitable for the power system with distributed BESS and can effectively improve its transient stability.

\section{System-level mathematical model of BESS}

In this paper, BESS adopts the structure of direct connection on the DC side. This structure has the characteristics of low energy consumption and easy control. The specific circuit topology is shown in Figure 1. It mainly includes elementary battery model, voltage source converter (VSC), isolation transformer and lowpass filter.

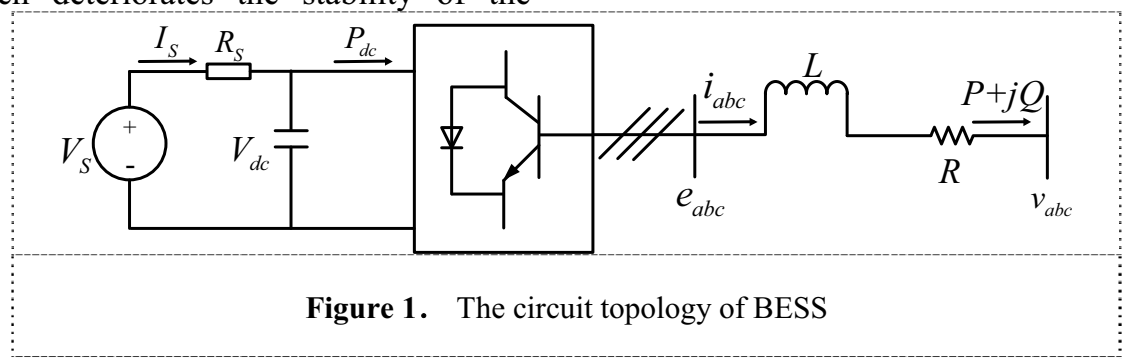

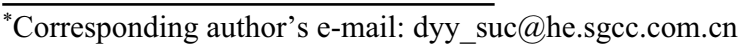




\subsection{Low-frequency mathematical model of VSC described by duty cycle}

In the abc three-phase static coordinate system, the fundamental frequency dynamic mathematical model on AC side of the VSC can be written in the following equations,

$$
\left[\begin{array}{l}
e_{a} \\
e_{b} \\
e_{c}
\end{array}\right]-\left[\begin{array}{c}
v_{a} \\
v_{b} \\
v_{c}
\end{array}\right]=R\left[\begin{array}{l}
i_{a} \\
i_{b} \\
i_{c}
\end{array}\right]+L \frac{d}{d t}\left[\begin{array}{l}
i_{a} \\
i_{b} \\
i_{c}
\end{array}\right]
$$

where, $v_{a}, v_{b}, v_{c}$ are the three phase $\mathrm{AC}$ voltages at the grid connection point, $e_{a}, e_{b}, e_{c}$ are the three phase output voltages of the converter, $R$ and $L$ are the equivalent resistance and capacitance of the isolation transformer and the low-pass filter.

The Park transform is used to convert the timevarying alternating currents in the abc three-phase stationary coordinate system into the steady direct currents in the dq0 coordinate system. With the phaselocked loop, the $\mathrm{d}$-axis of the synchronously rotating $\mathrm{dq} 0$ coordinate system is locked on $v_{d}$, that is, the angle between the d-axis and the voltage is $0, v_{d}=V, v_{q}=0$. The steady-state equations on the $\mathrm{AC}$ side of the VSC in the dq0 coordinate system are as follow.

$$
\left[\begin{array}{l}
e_{d} \\
e_{q}
\end{array}\right]-\left[\begin{array}{l}
V \\
0
\end{array}\right]=\left[\begin{array}{cc}
R & -\omega L \\
\omega L & R
\end{array}\right]\left[\begin{array}{l}
i_{d} \\
i_{q}
\end{array}\right]+L \frac{d}{d t}\left[\begin{array}{l}
i_{d} \\
i_{q}
\end{array}\right]
$$

Based on the instantaneous power theory, the active power and reactive power injected into the grid can be described as follow.

$$
\left\{\begin{array}{c}
P_{g}=\frac{3}{2}\left(v_{d} i_{d}+v_{q} i_{q}\right)=\frac{3}{2} V i_{d} \\
Q_{g}=\frac{3}{2}\left(v_{q} i_{d}-v_{d} i_{q}\right)=-\frac{3}{2} V i_{q}
\end{array}\right.
$$

In an $\mathrm{AC}$ system, $\mathrm{VSC}$ is equivalent to a voltage source whose phase and amplitude can be controlled. By adjusting the amplitude modulation ratio and initial phase angle of the PWM, the amplitude and phase of the modulation wave can be controlled, thereby achieving independently decoupling control of the voltage amplitude and phase of the VSC. The relationship between the VSC controlled quantities can be expressed as follow.

$$
\left[\begin{array}{l}
e_{d} \\
e_{q}
\end{array}\right]=M \frac{V_{d c}}{2}\left[\begin{array}{c}
\cos \delta \\
\sin \delta
\end{array}\right]
$$

\subsection{Mathematical model of BESS on DC side}

On the DC side, BESS adopts the elementary model, where $V_{S}$ is the output voltage of BESS, $R_{S}$ is the equivalent internal resistance, and $I_{S}$ is the output current of BESS.

$$
\left\{\begin{array}{c}
V_{d c}=V_{s}-I_{s} R_{s} \\
I_{d c}=\frac{V_{s}-V_{d c}}{R_{s}}-C \frac{d V_{d c}}{d t} \\
P_{d c}=V_{d c} I_{d c}
\end{array}\right.
$$

Regardless of the loss of the converter and the consumption of active power on the line, the energy on the DC side and the $\mathrm{AC}$ side are kept equal. The mathematical model of the DC side can be described as follow.

$$
\frac{V_{s}-V_{d c}}{R_{s}}-C \frac{d V_{d c}}{d t}=\frac{\frac{3}{2} V i_{d}}{V_{d c}}
$$

In summary, BESS mathematical model based on the rotating coordinate system can be expressed as follow.

$$
\left[\begin{array}{c}
\frac{V}{L} \\
0 \\
\frac{V_{S}}{R_{S} C}
\end{array}\right]=\left[\begin{array}{ccc}
-\frac{R}{L} & \omega & \frac{M \cos \delta}{2 L} \\
\omega & \frac{R}{L} & -\frac{M \sin \delta}{2 L} \\
\frac{3 M \cos \delta}{2 C} & \frac{3 M \sin \delta}{2 C} & \frac{1}{R_{S} C}
\end{array}\right]\left[\begin{array}{c}
i_{d} \\
i_{q} \\
V_{d c}
\end{array}\right]+\frac{d}{d t}\left[\begin{array}{c}
-i_{d} \\
i_{q} \\
V_{d c}
\end{array}\right]
$$

\section{VSC controller and its parameter optimization}

\subsection{Design of double closed-loop decoupling controller}

Based on the VSC mathematical model in the dq0 coordinate system, this paper proposes a double closedloop control strategy to independently control the d-axis voltage and q-axis voltage on the AC side of the VSC.

The double closed-loop control strategy includes an outer loop controller and an inner loop controller. The outer loop controller mainly includes voltage and current detection, sampling feedback, numerical calculation and the design of the corresponding regulator. Its outputs provide reference values for the inner loop controller. The inner loop controller includes decoupling block and the closed-loop control block. 


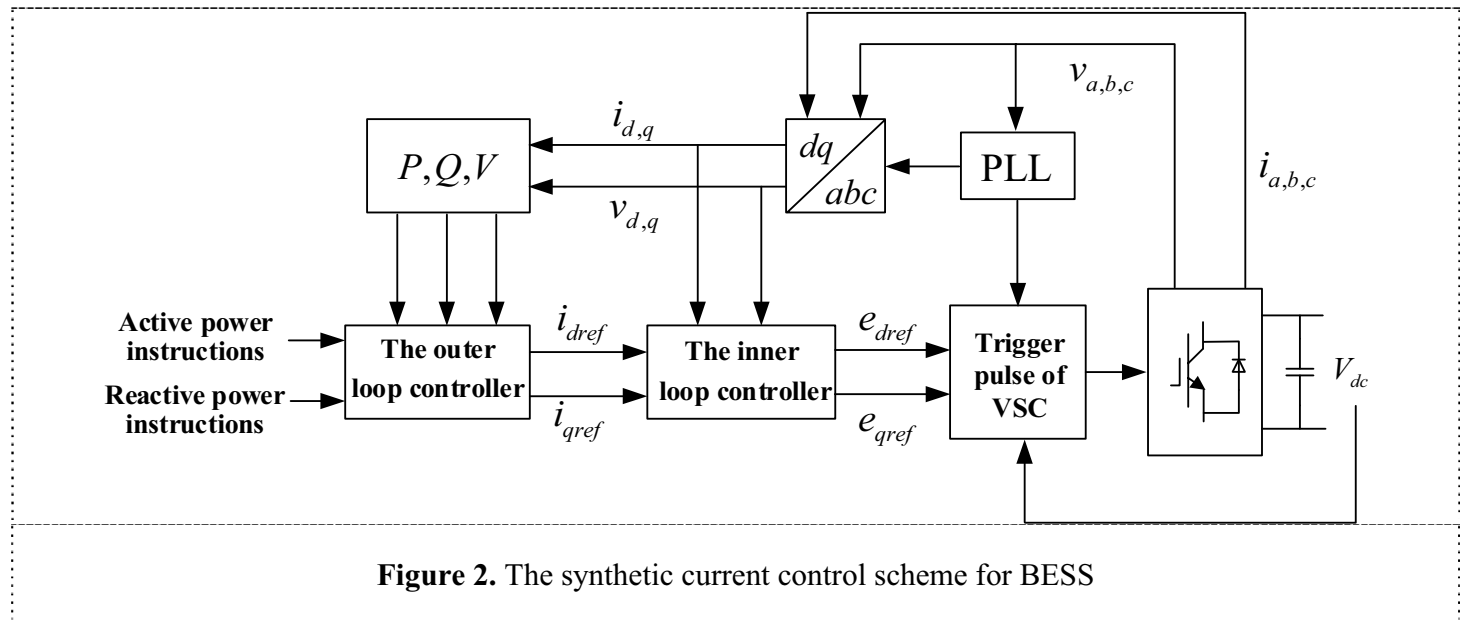

The outer loop inputs can be divided into:

a. Active power instructions are indicators directly related to active power, such as generator power angle difference and voltage phase angle difference.

b. Reactive power instructions are indicators directly related to reactive power, such as voltage amplitude.

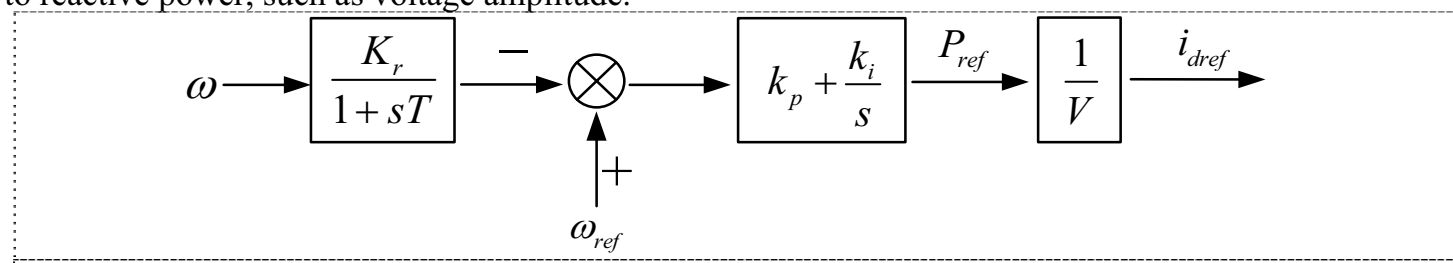

(a) Active power instruction and reference

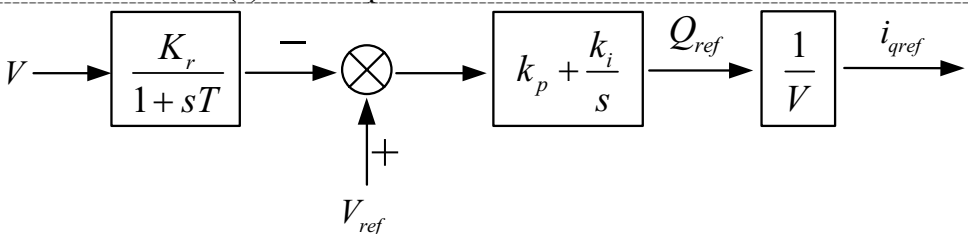

(b) Reactive power instruction and reference

Figure 3. The structure of the outer loop power controller

The inner loop control part adopts the current decoupling control strategy, so that the d-axis current $\boldsymbol{i}_{\boldsymbol{d}}$ and the q-axis current $\boldsymbol{i}_{\boldsymbol{q}}$ of the AC side current of the VSC track the current reference values $\boldsymbol{i}_{\text {dref }}$ and $\boldsymbol{i}_{\text {qref }}$ from the outer loop controller. Referring to equation (2), the inner loop controller can be described by the following equations.

$$
\left\{\begin{array}{c}
e_{d}=v_{d}+\left(k_{p 1}+k_{i 1}\right) \cdot\left(i_{d r e f}-i_{d}\right)-\omega L i_{q} \\
e_{q}=\left(k_{p 2}+k_{i 2}\right) \cdot\left(i_{\text {qref }}-i_{q}\right)+\omega L i_{d}
\end{array}\right.
$$

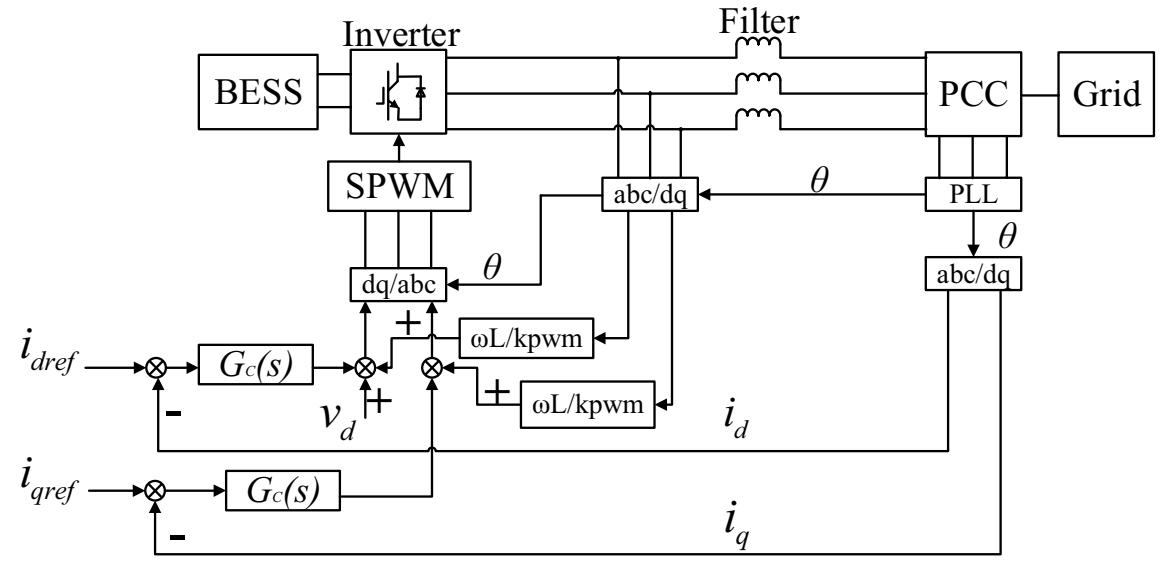

Figure 4. The structure of the inner loop power controller 


\subsection{PI controller parameters optimization using Particle swarm algorithm}

In order to coordinate the distributed BESS controllers, an optimization coordination strategy based on particle swarm optimization is proposed in this paper. Time domain dynamic performance indices are used as the objective function to optimize the controller parameters. The proposed algorithm has strong versatility and global search capabilities.

The basic principles of particle swarm optimization are as follows. Firstly, initialize a group of particles in the feasible solution space. Each particle represents a potential optimal solution of the optimization problem. The position, velocity, and fitness value are used to

represent the characteristics of each particle. In particular, the fitness value represents the particle's superiority.

The particles move in the solution space, and their individual positions are constantly updated by tracking the individual extreme value $\mathrm{P}$ and the group extreme value $G$. The individual extremum $P$ refers to the position with the optimal fitness value calculated in the positions experienced by the individual, and the collective extremum $G$ refers to the optimal position of the fitness searched by multiple particles in the population. The fitness value is calculated every time a particle updates its position, and the individual extreme value $\mathrm{P}$ and the group extreme value $\mathrm{G}$ are updated by comparing the fitness value of the new particle with the former $\mathrm{P}$ and $\mathrm{G}$.

The block diagram of the PSO algorithm is shown in Figure 5.

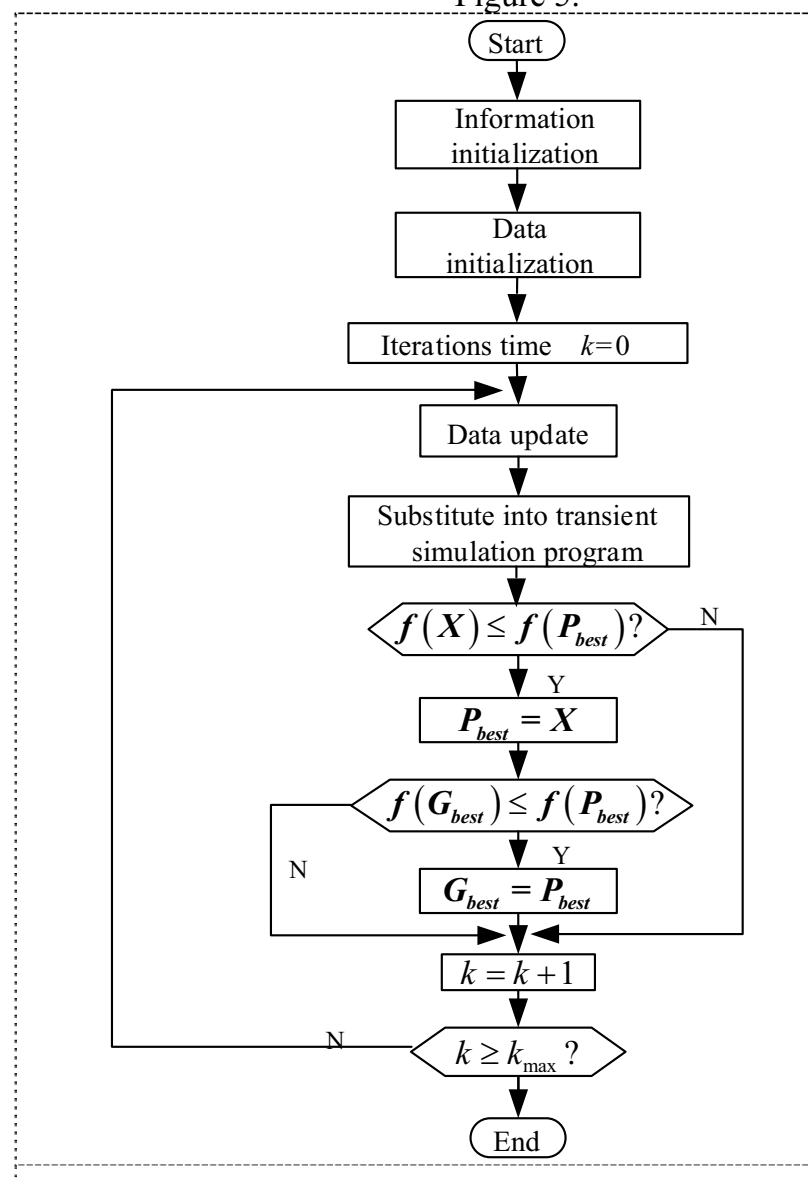

Figure 5. The flow chart for control parameters optimization

Suppose that in a D-dimensional search space, a population $\boldsymbol{X}=\left(\boldsymbol{X}_{1}, \boldsymbol{X}_{2}, \ldots \boldsymbol{X}_{\boldsymbol{n}}\right)$ composed of n particles. The i-th particle $\boldsymbol{X}_{\boldsymbol{i}}=\left(\boldsymbol{x}_{\boldsymbol{i} 1}, \boldsymbol{x}_{\boldsymbol{i} 2}, \ldots \boldsymbol{x}_{\boldsymbol{i}}\right)^{\boldsymbol{T}}$ represents the position of the $\mathrm{i}$-th particle in the search space, which is a potential solution of the problem. According to the objective function, the fitness value corresponding to each particle position can be calculated. For the i-th particle, its speed vector is $\boldsymbol{V}_{\boldsymbol{i}}=\left(\boldsymbol{V}_{\boldsymbol{i 1}}, \boldsymbol{V}_{\boldsymbol{i} 2}, \ldots \boldsymbol{V}_{\boldsymbol{i D}}\right)^{\boldsymbol{T}}$, its individual extreme value is $\boldsymbol{P}_{\boldsymbol{i}}=\left(\boldsymbol{P}_{\boldsymbol{i 1}}, \boldsymbol{P}_{\boldsymbol{i} 2}, \ldots \boldsymbol{P}_{\boldsymbol{i D}}\right)^{\boldsymbol{T}}$, and the extreme value of the population is $\boldsymbol{G}=$ $\left(G_{1}, G_{2}, \ldots G_{D}\right)^{T}$
During each iteration, the particle updates its own speed and position through individual extreme values and group extreme values.

$$
\begin{aligned}
& V_{i d}^{t+1}=\omega V_{i d}^{t}+c_{1} r_{1}\left(P_{i d}^{t}-X_{i d}^{t}\right)+c_{2} r_{2}\left(G_{d}^{t}-X_{i d}^{t}\right) \\
& X_{i d}^{t+1}=X_{i d}^{t}+V_{i d}^{t+1}
\end{aligned}
$$

To improve the transient stability of the system, the generator angle oscillation should be reduced and the period of oscillation time should be shortened. Therefore, the summation of the power angle difference integrals of the generators is selected as the objective function. 


$$
\min J=\int_{0}^{t} t \sum_{i=1}^{M}\left|\Delta \delta_{i}(t)\right| d t
$$

The objective function is subject to the following constraints.

$$
\text { s.t. }\left\{\begin{array}{l}
k_{p, g}^{P \min }<k_{p, g}^{P}<k_{p, g}^{P \max } \\
k_{i, g}^{P \min }<k_{i, g}^{P}<k_{i, g}^{P \max } \\
k_{p, g}^{\mathrm{Q} \min }<k_{p, g}^{Q}<k_{p, g}^{\mathrm{Q} \max } \\
k_{i, g}^{\mathrm{Q} \min }<k_{i, g}^{Q}<k_{i, g}^{\mathrm{Q} \max }
\end{array}\right.
$$

where, $\boldsymbol{k}_{\boldsymbol{p}, \boldsymbol{g}}^{\boldsymbol{P}}, \boldsymbol{k}_{\boldsymbol{i}, \boldsymbol{g}}^{\boldsymbol{P}}, \boldsymbol{k}_{\boldsymbol{p}, \boldsymbol{g}}^{\boldsymbol{Q}}$ and $\boldsymbol{k}_{\boldsymbol{i}, \boldsymbol{g}}^{\boldsymbol{Q}}$ are the PI control parameters of the g-th VSC controller, $\Delta \boldsymbol{\delta}_{\boldsymbol{i}}(\boldsymbol{t})$ is the power angle deviation of the i-th generator.

\subsection{Simulation examples}

The case discussed in this paper is based on the standard IEEE 3-machine 9-node system. The topology and detailed data of the system can be found in [10]. Since the load of the 3-machine 9-node system is basically evenly distributed near the Bus 5, Bus 6 and Bus 8, the distributed BESS is also evenly allocated, that is, three groups of BESS with a capacity of $10 \mathrm{MW}$ are respectively connected to Bus 5, Bus 6 and Bus 8. Each distributed BESS controller adopts a double closed-loop decoupling control strategy, and the PSOt toolbox is used to optimize and coordinate the parameters of the distributed controllers.

Assuming that a three-phase short-circuit fault occurs at the end of a Line 4 at $\mathrm{t}=1 \mathrm{~s}$. After $0.1 \mathrm{~s}$, the three-phase circuit breakers trip to clear the fault. After another $0.5 \mathrm{~s}$, the circuit breakers reclose, and the system restores to the topology before the failure.

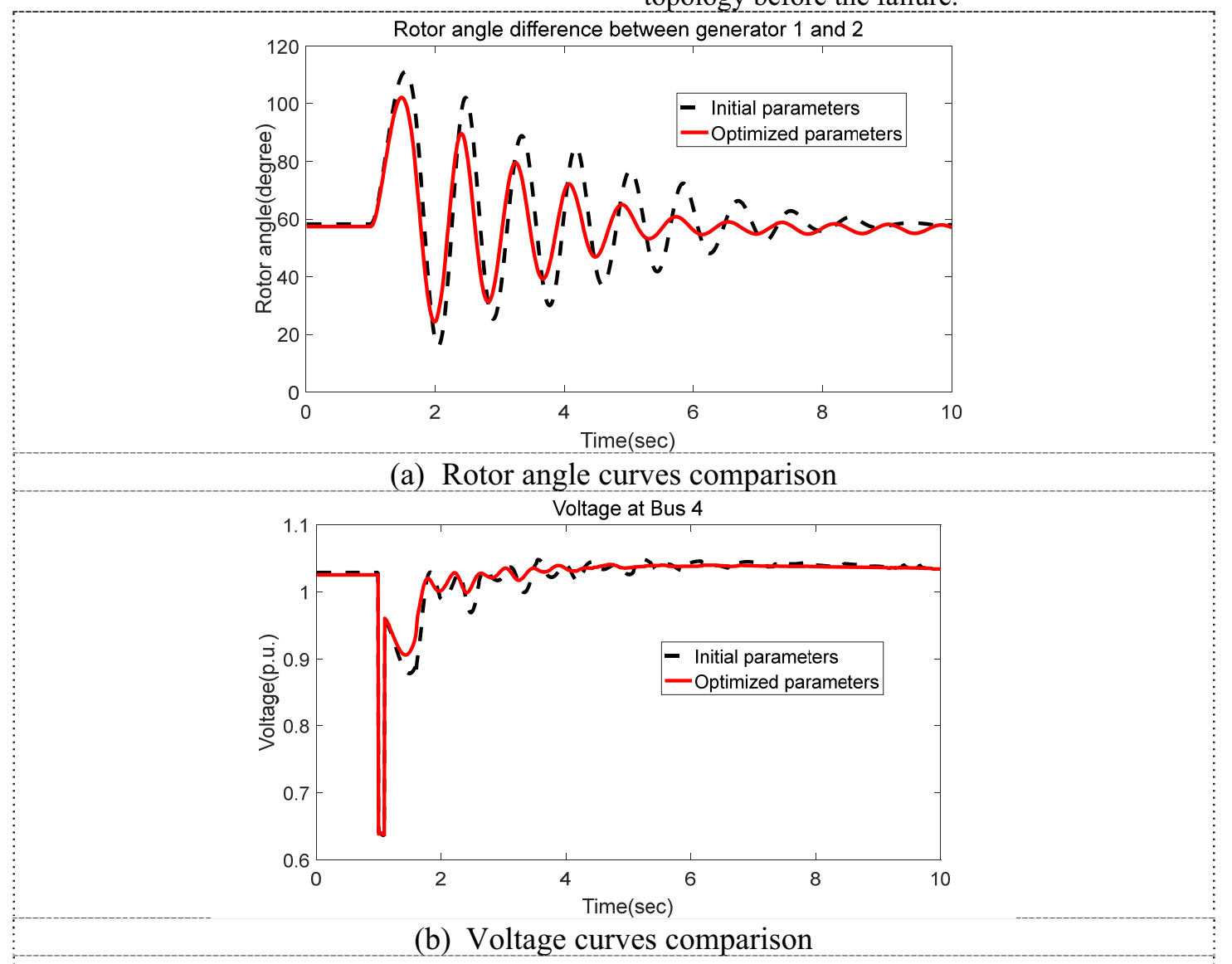

Figure 6. Simulation results for fault at line 4

Simulation results are shown in Figure 6. Compared with the initial parameters, the distributated controllerd with optimized parameters can improve the transient stability of the system to a greater extent.

\section{Conclusions}

This paper proposes a new method to improve the transient stability of power system by using distributed BESS. Considering the interaction between multiple BESS controllers, this paper proposes a method based on particle swarm optimization to ensure the coordination between controllers. Simulation results show that the coordinated control method proposed in this paper is suitable for the power system with distributed BESS and can effectively improve the dynamic performance of the system. 


\section{Acknowledgments}

This work was supported in part by Electric Power Research Institute of State Grid Hebei Electric Power Company under grant kj2020-083.

\section{References}

1. Chakraborty A, Musunuri S K, Srivastava A K, et al. Integrating STATCOM and Battery Energy Storage System for Power System Transient Stability: A Review and Application[J]. Advances in Power Electronics, 2012, 2012(676010): 1-12.

2. Xi Z, Parkhideh B, Bhattacharya S. Improving distribution system performance with integrated STATCOM and supercapacitor energy storage system[C]. IEEE Power Electronics Specialists Conference. IEEE, 2008:1390-1395.

3. Liu J., Fang W., Zhang X., Yang C.. An improved photovoltaic power forecasting model with the assistance of aerosol index data[J]. IEEE Transactions on Sustainable Energy, 2015, 6(2): 434-442.

4. Beza M, Bongiorno M. An Adaptive Power Oscillation Damping Controller by STATCOM With Energy Storage[J]. IEEE Transactions on Power Systems, 2015, 30(1): 484-493.

5. Castro L M, Acha E, Fuerte-Esquivel C R. A Novel STATCOM Model for Dynamic Power System Simulations[J]. IEEE Transactions on Power Systems, 2013, 28(3): 3145-3154.

6. Song Q, Liu W, Yuan Z. Multilevel Optimal Modulation and Dynamic Control Strategies for STATCOMs Using Cascaded Multilevel Inverters[J]. IEEE Transactions on Power Delivery, 2007, 22(3): 1937-1946.

7. Gavriluta C, Candela J I, Rocabert J, et al. Adaptive Droop for Control of Multiterminal DC Bus Integrating Energy Storage[J]. IEEE Transactions on Power Delivery, 2015, 30(1): 16-24.

8. Liu, J., Su, C., Wang, X., et al. Abnormality in Power System Transient Stability Control of BESS/STATCOM[C]. IET RPG2017, Wuhan, China, Oct. 19 - 20, 2017: 1-6.

9. Ding K., Liu J., Wang X., Zhang X., Wang N.. "Research of active and reactive power coordinated control method for photovoltaic inverters based on an improved double-loop control scheme", 2016 China International Conference on Electricity Distribution (CICED 2016), Xi'an, China, Aug. 1013, 2016: 1-5.

10. Wang X. Modern Power System Analysis [M]. Science Press, 2009. 